\title{
Prostaglandin E2 EP4 Receptor Activation Attenuates Neuroinflammation and Early Brain Injury Induced by Subarachnoid Hemorrhage in Rats
}

\author{
Jie $\mathrm{Xu}^{1} \cdot \mathrm{Zhen} \mathrm{Xu}^{2} \cdot \mathrm{Ai} \operatorname{Yan}^{1}$
}

Received: 13 September 2016 / Revised: 19 December 2016 / Accepted: 26 December 2016 / Published online: 27 February 2017 (c) The Author(s) 2017. This article is published with open access at Springerlink.com

\begin{abstract}
Activation of E prostanoid 4 receptor (EP4) shows neuroprotective effects in multiple central nervous system (CNS) lesions, but the roles of EP4 receptor in subarachnoid hemorrhage (SAH) are not explored. This study was designed to research the effects of EP4 modulation on early brain injury (EBI) after experimental SAH in rats. We found that the administration of EP4 selective agonist AE1329 significantly improved neurological dysfunction, blood brain barrier (BBB) damage and brain edema at $24 \mathrm{~h}$ after SAH. Furthermore, AE1-329 obviously reduced the number of activated microglia and the mRNA and protein levels of pro-inflammatory cytokines, and increased Ser1177 phosphorylated endothelial nitric oxide synthase (Ser1177 p-eNOS). Moreover, AE1-329 significantly reduced the number of TUNEL-positive cells and active caspase-3 in cortex after SAH. The EP4 selective antagonist AE3208 was also administrated and the opposite effects were achieved. Our results indicate that activation of EP4 protects brain from EBI through downregulating neuroinflammation reaction after $\mathrm{SAH}$.
\end{abstract}

Ai Yan

yanai71@163.com

Jie Xu

xujiexujie81@163.com

Zhen Xu

xuzhenhangzhou@163.com

1 Department of Neurosurgery, Huzhou Central Hospital, 198 Hongqi Lane, Huzhou 313003, China

2 Department of Neurosurgery, First Affiliated Hospital of Zhejiang Chinese Medicine University, 54 Youdian Lane, Hangzhou 310006, China
Keywords EP4 receptor - Inflammation - Early brain injury $\cdot$ Subarachnoid hemorrhage

\section{Introduction}

Subarachnoid hemorrhage (SAH) is one of the most devastating cerebrovascular diseases with high morbidity and mortality, and often results in long lasting neurological disability for survivors [1]. Despite the advances in neurointensive care, the underlying mechanisms of SAH-induced secondary brain injury are still incompletely understood. During the past decades, research efforts have been centered around vasospasm which is considered to be the most important factor for delayed neurological deficit [2, 3]. However the long lasting failure of anti-vasospastic treatments to improve outcome of SAH in most clinical trials [4] has brought into focus the significance of a more recently found pathological phenomenon which named early brain injury (EBI) [5]. EBI refers to the global brain injury which starts immediately after SAH and lasts $72 \mathrm{~h}$ until the development of vasospasm [6, 7]. Evidences suggest that SAH-induced EBI is not only responsible for the initial signs and symptoms on admission, but also blamed for the delayed neurological deterioration which is associated with vasospasm and poor long-term prognosis [8, 9]. The underlying pathophysiological mechanisms of EBI after SAH are not definitely clarified to date. It is generally accepted that neuroinflammation plays a critical role in the happening and progressing of EBI after SAH [10-12].

Microglial cell is the resident macrophage of the central nervous system (CNS). After SAH, especially in acute stage, activated microglias provoke excessive secretion of pro-inflammatory cytokines contributing to the development of brain edema, disruption of blood-brain barrier 
(BBB), and secondary neuronal injury after SAH [5]. The pro-inflammatory cytokines secreted by activated microglial cells include interleukin-6 (IL-6), interleukin-1 beta (IL-1 $\beta$ ) and tumor necrosis factor alpha (TNF- $\alpha$ ) [13] all of which have been found to be increased early after SAH and strongly linked to the brain injury in both patients and animals [14-16]. Thus, modulating the inflammatory cytokine secretion of activated microglial cells might be a potential strategy for the treatment of EBI after SAH.

The lipid signaling molecule prostaglandin E2 (PGE2), which is one of the most typical downstream products of arachidonic acid (AA) by cyclooxygenases- 1 and 2 (COX-1 and 2), is a well-established modulator of inflammatory responses in a variety of CNS and peripheral injury models [17]. PGE2 modulates inflammatory responses through activating four distinct $\mathrm{G}$ protein-coupled receptors (GPCRs) named E prostanoid 1-4 receptors (EP 1-4 receptors), which exhibit divergent cellular expression profiles, desensitization kinetics and signaling cascades [18, 19]. Among them the EP4 receptor is emerging as the most promising and versatile one and the effects of anti-thrombosis, anti-inflammation and vasodilation have been proposed for it [20]. In vivo and in vitro studies have proved that activation of EP4 receptor by exogenous EP4 selective agonist suppresses microglial inflammatory response to $A \beta_{42}$ peptides and lipopolysaccharide while conditional deletion of microglial EP4 conversely increases inflammatory gene expression [21, 22]. A broad variety of experimental neuropathological models associated with inflammation are also alleviated by the activation of EP4 receptor, including cerebral ischemia [23, 24], hypoxic-ischemic encephalopathy (HIE) [25], neurotoxicity induced brain injury [26] and Alzheimer's disease [21].

The roles of EP4 receptor in EBI after SAH are currently unknown. Based on these prominent studies, it is reasonable to deduce that the activation of EP4 receptor might suppress microglial activation as well as microglia-induced neuroinflammation and thus play a protectional role in EBI after $\mathrm{SAH}$

In this study, we investigated the effects of EP4 receptor activation on the microglial activation, neuroinflammation and EBI after SAH by selectively using EP4 receptor specific agonist or antagonist in a rat model.

\section{Materials and Methods}

\section{Animals}

Experimental animal was male Sprague-Dawley rats (280-350 g), afforded by animal experimental center of Zhejiang Chinese Medicine University. The use and care of animals employed in our model were approved by the
Animal Care and Use Committee of Zhejiang Chinese Medicine University, in accordance with all relevant laws of China.

All animals were kept in a room with controlled temperature of $23 \pm 1{ }^{\circ} \mathrm{C}$ at 12 -h dark/light cycle and fed with standard food and water ad libitum.

\section{Rat Model of SAH}

An endovascular perforation method was used to produce the model of experimental SAH according to previous studies $[27,28]$. Briefly, after anesthetizing with the mixture of 3\% isoflurane in 70\%/30\% medical air/oxygen, the right external carotid artery was transected distally and then reflected caudally in line with the internal carotid artery. A blunted monofilament nylon suture of 4-0 was inserted into the right external carotid artery stump and then carefully advanced into the internal carotid artery until a resistance was felt, approximately $20-24 \mathrm{~mm}$ from the common carotid artery bifurcation. The suture was advanced for another $3 \mathrm{~mm}$ to perforate the right internal carotid artery close to the bifurcation with the middle cerebral artery to produce SAH. Sham-operated animals were suffered the similar procedures except the arterial perforation.

After operation, the rats were returned to their cages with the room temperature kept at $23 \pm 1{ }^{\circ} \mathrm{C}$. Physical cooling (ice bag) was used to keep the rectal temperature at $37 \pm 0.5^{\circ} \mathrm{C}$ when required.

\section{Drug Administration}

The EP4 receptor selective agonist AE1-329 and antagonist AE3-208 were from Ono Pharmaceuticals Co (Japan). Their specificity for the EP4 receptor has been proved previously $[29,30]$. AE $1-329(0.3 \mathrm{mg} / \mathrm{kg})$ was subcutaneously injected (SC) [23]. In several models of cerebral diseases, AE3-208 is administrated per os to reach an effective concentration in brains $[31,32]$. Based on these studies, we administrated AE3-208 $(0.3 \mathrm{mg} / \mathrm{kg})$ subcutaneously. AE1329 and AE3-208 were dissolved in a solution consisting of 5\% $N$-methyl pyrrolidone (NMP) and 5\% solutol HS in saline, and each rat was subcutaneously injected with $2 \mathrm{ml} /$ kg AE1-329, AE3-208 or vehicle (5\% $N$-methyl pyrrolidone (NMP) and 5\% solutol HS in saline).

All drugs were administrated immediately after SAH and were repeated $12 \mathrm{~h}$ post SAH with the same dose.

\section{Assessment of SAH Grade, Mortality and Neurological Score}

The methods of SAH grade [33] and neurological score $[34,35]$ evaluation were described by previous studies and 
all evaluations were performed by an experimenter blinded to this study.

Twenty-four hours after SAH, rats were sacrificed after anesthetization and brains were removed immediately for the measurement of SAH grades. SAH grade was evaluated according to the amount of subarachnoid blood clots distributed in the six segments of basal cistern: grade one (scores $=0$ ), no observable subarachnoid blood clots; grade two (scores $=1$ ), minimal subarachnoid blood clots; grade three (scores $=2$ ), moderate subarachnoid blood clots with recognizable arteries; and grade four (scores $=3$ ), subarachnoid blood clots covering all arteries. Possible scores ranged from 0 to 18 were calculated by the total scores from all six segments. The animal's neurological deficits were evaluated by six aspects: spontaneous activity (scores ranged from 0 to 3 ), reaction to side stroking (scores ranged from 1 to 3), vibrissae touch (scores ranged from 1 to 3 ), forepaw outstretching (scores ranged from 0 to 3), climbing (scores ranged from 0 to 3 ), limb symmetry (scores ranged from 0 to 3 ), and beam walking ability which refers to the walking distances on a wooden beam for $1 \mathrm{~min}$ (scores ranged from 0 to 4 ). The minimum score of neurological deficits was 2 and the maximum was 22; a higher score represent better neurological performance. Mortality was calculated at the same time.

\section{Assessment of Brain Edema}

Twenty-four hours after SAH, rats were sacrificed after anesthetization and entire brains were removed immediately for the measurement of water content [36]. Water content of brain was determined by the formula (wet weight - dry weight)/(wet weight $) \times 100 \%$. The wet weight of brains was obtained by the weight of freshly removed brains, then the wet brains were kept in an oven for $72 \mathrm{~h}$ at $100^{\circ} \mathrm{C}$ and weighed to obtain dry weight.

\section{Blood Brain Barrier Permeability}

BBB permeability was determined by Evans blue (EB) extravasation $24 \mathrm{~h}$ after SAH. Briefly, 2\% Evans blue dye was injected in 2 min into the right femoral vein at a dose of $2 \mathrm{ml} / \mathrm{kg}$, allowing the dye to circulate for a total of 60 min [37]. Animals were re-anesthetized and subjected to transcardial perfusion with phosphate buffered saline (PBS) to remove intravascular EB dye, then entire brains were removed and homogenized in phosphate buffered saline. Trichloroacetic acid was then added to precipitate protein. After overnight incubation at $4{ }^{\circ} \mathrm{C}$, samples were centrifuged and the resulting supernatants were measured for absorbance of EB at $620 \mathrm{~nm}$ using a spectrophotometer.

\section{TUNEL Staining}

Twenty-four hours after SAH, rats were anesthetized and perfused transcardially with $4 \%$ paraformaldehyde. Brains were separated and fixed in $4 \%$ paraformaldehyde for $24 \mathrm{~h}$ and dehydrated with gradient sucrose solution. Consecutive coronal sections of $10 \mu \mathrm{m}$ were cut from bregma with $200 \mu \mathrm{m}$ intervals. A total of 8 sections in right hemisphere of brain (perforation side) were obtained for terminal deoxynucleotidyl transferase-mediated dUTP-biotin nick end labeling (TUNEL) staining (Roche Diagnostics, Mannheim, Germany). DAPI staining was performed according to the instructions. The images were viewed with an EVOS-fl digital inverted fluorescent microscopy and five non-overlapped vision fields were randomly chosen for observation in each section. The number of TUNEL positive cells was counted with Image Pro Plus 6.0 Software (MediaCybernetics, Bethesda, USA). The mean number of TUNEL/ DAPI double positive nuclei in the five views was taken for the apoptotic index of each section and the apoptotic index for each animal was calculated by the final average percentage of double positive cells of the 8 sections.

\section{Western Blot}

The cortex and cerebral microvessels of right hemisphere were separated for western blot analysis. Samples were incubated with protease inhibitor (Roche) then diluted with sample buffer. Samples were supplemented with $2 \%$ beta-mercaptoethanol and $50 \mathrm{mM}$ DTT and boiled for about $5 \mathrm{~min}$. Proteins were separated on tris-glycine 4-15\% acrylamide gels and transferred to PVDF membranes soaked in 5\% nonfat milk in PBS-Tween $20(0.05 \%)$ for $2 \mathrm{~h}$. The proteins of total caspase-3, cleaved caspase-3, Ser1177 phosphorylated endothelial nitric oxide synthase (Ser1177 p-eNOS) and $\beta$-actin were tested by rabbit monoclonal antibody against caspase-3 (Santa Cruz, 1:500), rabbit polyclonal antibody against cleaved caspase-3 (Biorbyt, 1:500), rabbit polyclonal antibody against Ser 1177 p-eNOS (Invitrogen, 1:400) and rabbit polyclonal antibody against $\beta$-actin (Santa Cruz, 1:500) respectively. Immunoreactivity was tested by incubation with secondary HRP-coupled antibody for $1 \mathrm{~h}$ at room temperature followed by the ECL plus reagent (Santa Cruz). The densities of the bands were determined by the MiVnt image analysis system (Bio-Rad, Carlsbad, CA, USA).

\section{Immunofluorescence Staining}

Consecutive coronal sections of $10 \mu \mathrm{m}$ were cut from bregma with $200 \mu \mathrm{m}$ intervals. A total of eight sections in right hemisphere (perforation side) were obtained for immunofluorescence staining. The sections were incubated 
with rabbit polyclonal antibody against Iba-1 (Santa Cruz, $1: 300)$ at $4{ }^{\circ} \mathrm{C}$ overnight, then followed by incubating with Dylight 488-conjugated goat anti-rabbit antibody (Jackson ImmunoResearch, 1:1000). DAPI was used for nuclear staining. Sections viewed under the confocal microscope (Nikon). The number of Iba-1 positive cells was counted with Image Pro Plus 6.0 Software (MediaCybernetics, Bethesda, USA).

\section{Enzyme Linked Immunosorbent Assay}

The right hemisphere of brain was separated for the determining of the levels of inflammatory factors by a ELISA kits according to the instructions (TNF- $\alpha$ from Diaclone Research, Besancon, France; IL-1 $\beta$, IL-6 from BioSource Europe SA, Nivelles, Belgium).

\section{Quantitative Real-Time Polymerase Chain Reaction (RT-qPCR)}

The quantification of mRNA levels of inflammatory factors was performed by RT-qPCR as previously described [38]. The right hemisphere of brain (perforation side) was obtained at $24 \mathrm{~h}$ after SAH and total RNA from the cortex was isolated using TRIzol (Invitrogen, USA) with the PureLink RNA Mini Kit (Invitrogen, USA) as instructions. The purity and concentration of RNA were tested by spectrophotometric analysis (OD 260/280) and agarose gel electrophoresis. Then RNA was reverse-transcribed to obtain cDNA using SuperScript II Reverse Transcriptase (Invitrogen, USA). The reaction was ended by heating at $70^{\circ} \mathrm{C}$ for 10 min. RT-qPCR was performed using the CFX96 Realtime PCR detection system (Bio-Rad, USA), applying realtime SYBR Green PCR technology. The reaction mixtures $(25 \mu \mathrm{l})$ contained $12.5 \mu \mathrm{l}$ SYBR Green, $9.5 \mu \mathrm{l}$ nucleasefree water, $1 \mu \mathrm{l}$ cDNA, $1 \mu \mathrm{l}$ forward primer $(10 \mu \mathrm{M})$ and $1 \mu \mathrm{l}$ reverse primer $(10 \mu \mathrm{M})$. The primers sequences were designed by the Genscript Biological Technology Company (Nanjing, China) according to the gene sequences reported in GenBank. The IL-1 $\beta$ primers were $50^{\prime}$-CAC CTCTCAAGCAGAGCACAG-30' (forward) and 50'-GGG TTCCATGGTGAAGTCAAC-30' (reverse). The IL-6 primers were 50'-TCCTACCCCAACTTCCAATGCTC-30' (forward) and 50'-TTGGATGGTCTTGGTCCTTAGCC$30^{\prime}$ (reverse). The TNF- $\alpha$ primer were $50^{\prime}$-AAATGGGCT CCCTCTCATCAGTTC-30' (forward) and 50'-TCTGCT TGGTGGTTTGCTACGAC-30' (reverse). The GAPDH primers were 5'-ACAGCAACAGGGTGGTGGAC-3' (forward) and 5'-TTTGAGGGTGCAGCGAACTT-3' (reverse). After $95^{\circ} \mathrm{C}$ for $1 \mathrm{~min}, 40$ cycles were performed and each cycle contained a denaturation step at $95^{\circ} \mathrm{C}$ for $15 \mathrm{~s}$ and an annealing step at $60^{\circ} \mathrm{C}$ for $1 \mathrm{~min}$. The melting curve which was measured immediately after amplification, showed a single product peak indicating the good product specificity. All samples were analyzed in triplicate. Data were analyzed using the Line-Gene software. The mRNA expression levels were calculated with the $2-\Delta \Delta \mathrm{Cq}$ method and normalized by quantity of GAPDH mRNA.

\section{Statistic Analysis}

All data were presented as means \pm standard error of the mean (SEM) and SPSS 12.0 was used for statistical analysis of the results. Data were subjected to one-way analysis of variance (ANOVA) followed by the LSD and Dunnett's post-hoc test for multiple comparisons. The difference of mortality rate was analyzed by Chi square test. A value of $\mathrm{p}<0.05$ was considered as statistical significance.

\section{Results}

\section{Effects of EP4 Receptor on SAH Grade, Mortality and Neurological Score}

Twenty-four hours after SAH, the SAH grade of SAH group treated with vehicle $(14.4 \pm 1.41)$ did not significantly differ from those of treated with EP4 receptor specific agonist AE1-329 (13.8 \pm 1.36$)$ or antagonist AE3-208 $(14.1 \pm 1.38)$, suggesting the modulation of EP4 receptor by AE1-329 or AE3-208 did not affect the bleeding levels (Fig. 1b).

There are no animals of sham group died after surgery. The mortality of the SAH treated with vehicle was $32.1 \%$ ( 9 of 28 rats). The mortality of the SAH treated with AE1329 and AE3-208 was 21.4\% (6 of 28 rats) and 39.3\% (11 of 28 rats) respectively (Fig. 1c).

The neurological score was significantly decreased in the SAH group treated with vehicle $(12.8 \pm 1.3)$ compared with the sham group (20.8 \pm 1.5$)$. AE1-329 administration increased neurological score $(16.3 \pm 1.6)$ compared with SAH treated with vehicle, while AE3-208 showed opposite effects $(9.2 \pm 0.9)$ (Fig. 1d).

\section{Effects of EP4 Receptor on Brain Edema and BBB Permeability After SAH}

Twenty-four hours after SAH, brain water content of SAH group treated with vehicle $(81.47 \pm 0.22 \%)$ was significantly increased compared with sham group $(78.77 \pm 0.21 \%)$. AE1-329 decreased brain water content $(80.25 \pm 0.22 \%)$ while AE3-208 increased the brain water content $(82.63 \pm 0.23 \%)$ (Fig. 2a). Rats of SAH group treated with vehicle showed a significant increase in BBB permeability to Evans blue (19.9 $\mathrm{ng} / \mathrm{mg}$ protein) indicating an early damage of BBB after SAH. AE1-329 improved 
A

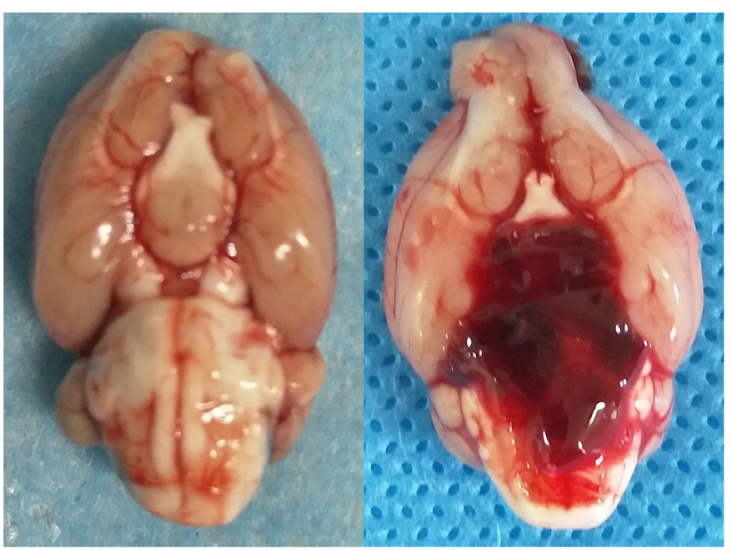

C

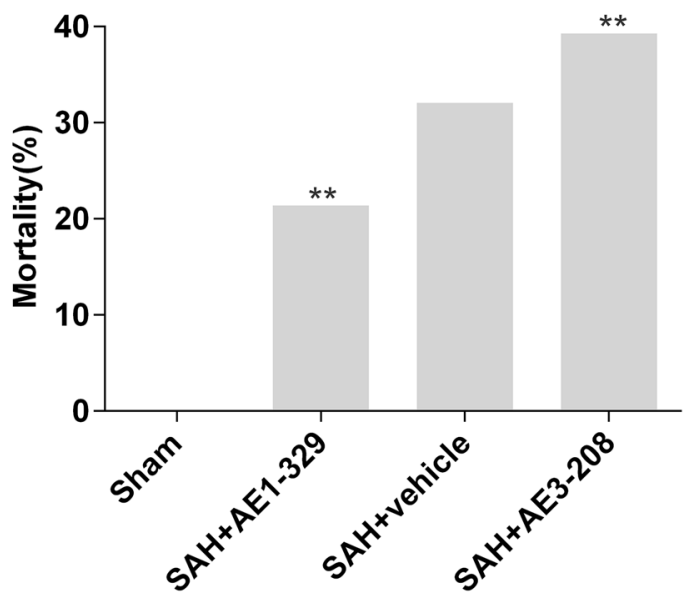

Fig. 1 Effects of EP4 receptor on SAH grade, mortality and neurological score $24 \mathrm{~h}$ after SAH. Representative images showed rat brains of sham and SAH groups (a). EP4 modulation did not affect the SAH grade $(n=12)$ (b). The activation of EP4 by AE1-329 decreased mortality $(n=28)$ and attenuated neurological deficits

the BBB damage as demonstrated by the decrease of Evans blue extravasation (15.6 $\mathrm{ng} / \mathrm{mg}$ protein) while AE3-208 showed opposite effects (23.8 $\mathrm{ng} / \mathrm{mg}$ protein) (Fig. 2b).

\section{Effects of EP4 Receptor on Neuronal Apoptosis After SAH}

Twenty-four hours post SAH, about $5.64 \pm 0.51 \%$ cells of SAH group treated with vehicle showed apoptosis (Fig. 4a, b). AE1-329 significantly mitigated cellular apoptosis $(3.12 \pm 0.29 \%)$ while AE3-208 aggravated cellular apoptosis $(9.39 \pm 0.97 \%)$ (Fig. 3a, b). The expression of cleaved caspase-3 in SHA group also increased dramatically

\section{B}
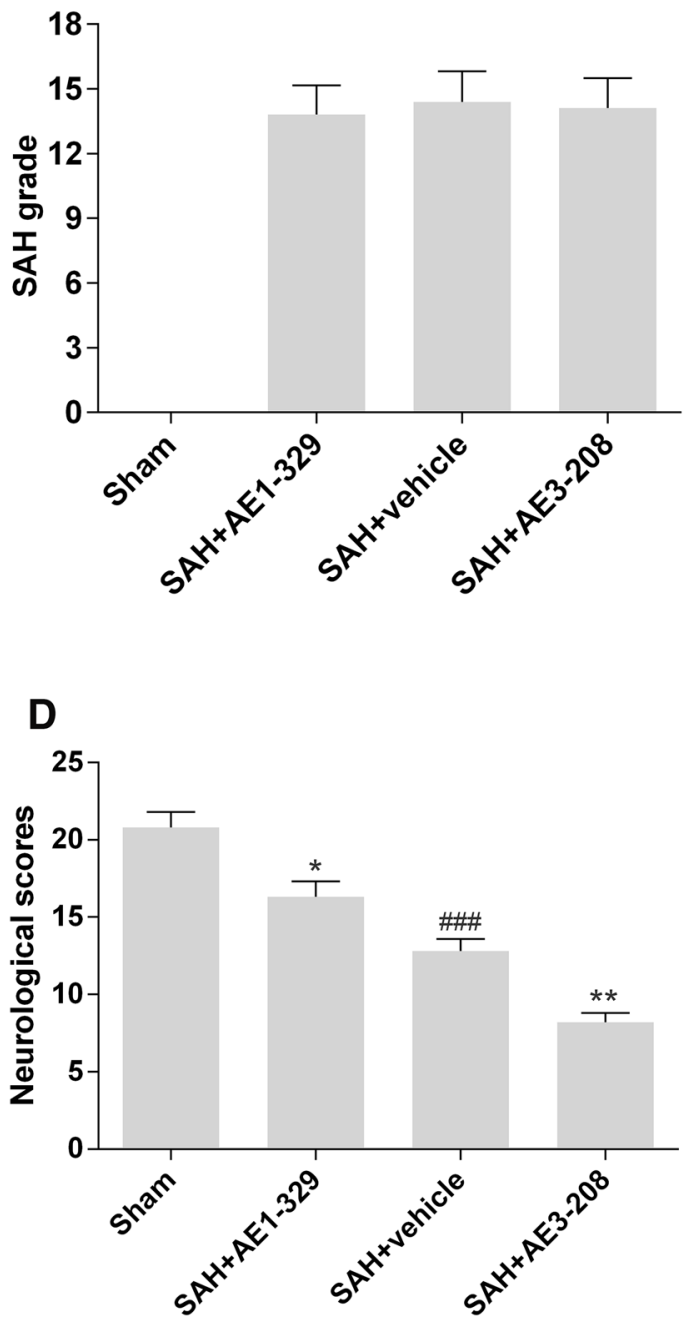

$(n=12) 24 \mathrm{~h}$ after SAH while the inhibition of EP4 by AE3-208 showed opposite effects $(\mathbf{c}, \mathbf{d})$. Data were expressed as mean \pm SEM $\left({ }^{\# \#} \mathrm{p}<0.001\right.$ vs. Sham, $* \mathrm{p}<0.05$ vs. SAH treated with vehicle, $* * \mathrm{p}<0.01$ vs. SAH treated with vehicle)

compared with that of sham group, which was downregulated by AE1-329 and upregulated by AE3-208 (Fig. 3c).

\section{Effects of EP4 Receptor on the Activation of Microglia After SAH}

After SAH, microglia transform from the resting status to highly activated status. The hallmarks of activated microglia include morphological changes, production of pro-inflammatory cytokines and enhanced proliferation [39-41].

In sham group, the density of Iba- 1 positive cells was $24.3 \pm 2.47 / \mathrm{mm}^{2}$. Twenty-four hours post $\mathrm{SAH}$, the microglias in SAH treated with vehicle group increased in 
A

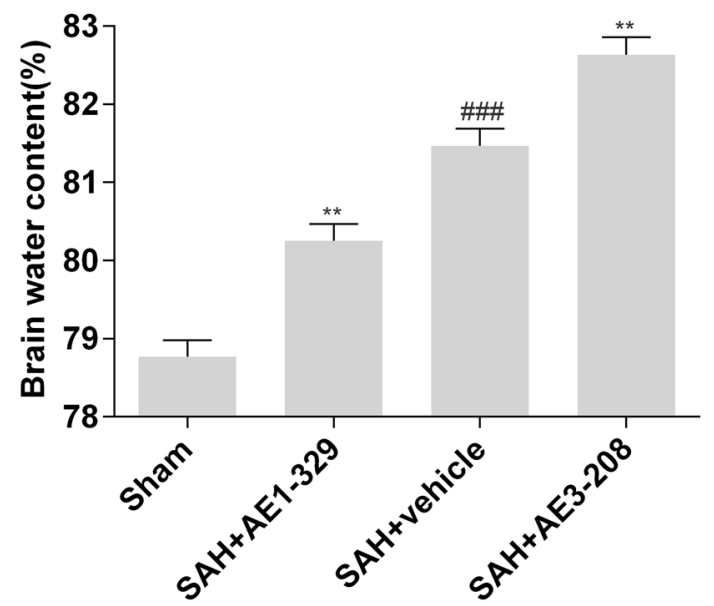

Fig. 2 Effects of EP4 receptor on brain edema and BBB permeability after SAH. The activation of EP4 by AE1-329 decreased brain water content and BBB permeability to Evans blue $24 \mathrm{~h}$ after SAH while the inhibition of EP4 by AE3-208 showed opposite effects $(\mathbf{a}, \mathbf{b})$.

number $\left(186.5 \pm 10.7 / \mathrm{mm}^{2}\right)$ and became activated, altering their morphology with upregulation of Iba-1 (Fig. 4a, b). AE1-329 significantly attenuated Iba-1 positive microglia number $\left(105.8 \pm 8.5 / \mathrm{mm}^{2}\right)$ and it's activation, while AE3208 showed opposite effects $\left(225.5 \pm 12.4 / \mathrm{mm}^{2}\right)$ (Fig. $4 \mathrm{a}$, b).

\section{Effects of EP4 Receptor on the Levels of Inflammatory Cytokines After SAH}

To further explore the effects of EP4 receptor activation on the neuroinflammation after SAH, we measured the mRNA and protein levels of pro-inflammatory cytokines TNF-a, $\mathrm{IL}-1 \beta$ and IL- 6 in the cortex at $24 \mathrm{~h}$ after injury. RT-qPCR analysis showed AE1-329 reduced the mRNA expression of TNF-a, IL-1 $\beta$ and IL-6 while AE3-208 increased the mRNA expression (Fig. 5a, c, e).

Enzyme linked immunosorbent assay showed, compared with the sham group $(5.9 \pm 0.27 \mathrm{ng} / \mathrm{g}$ in TNF$\alpha, 22.1 \pm 1.1 \mathrm{ng} / \mathrm{g}$ in IL- $1 \beta$ and $42.8 \pm 2.2 \mathrm{ng} / \mathrm{g}$ in IL-6 respectively), the levels of inflammatory cytokines significantly increased in the SAH treated with vehicle group $(16.9 \pm 0.85 \mathrm{ng} / \mathrm{g}$ in TNF- $\alpha, 57.0 \pm 2.3 \mathrm{ng} / \mathrm{g}$ in IL- $1 \beta$ and $81.4 \pm 3.9 \mathrm{ng} / \mathrm{g}$ in IL-6 respectively) $24 \mathrm{~h}$ after SAH. AE1329 downregulated the levels of inflammatory cytokines $(12.1 \pm 0.54 \mathrm{ng} / \mathrm{g}$ in TNF- $\alpha, 39.7 \pm 1.9 \mathrm{ng} / \mathrm{g}$ in IL- $1 \beta$ and $63.7 \pm 3.5 \mathrm{ng} / \mathrm{g}$ in IL-6 respectively) while AE3208 showed opposite effects $(21.3 \pm 1.1 \mathrm{ng} / \mathrm{g}$ in TNF- $\alpha$, $73.4 \pm 4.1 \mathrm{ng} / \mathrm{g}$ in IL- $1 \beta$ and $94.5 \pm 4.6 \mathrm{ng} / \mathrm{g}$ in IL-6 respectively) (Fig. 5b, d, f).
B

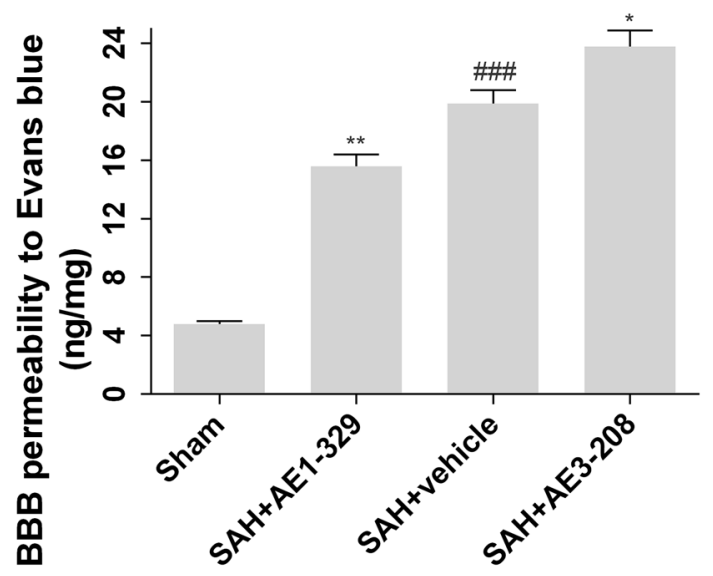

Data were expressed as mean \pm SEM $\left(n=12,{ }^{\# \# \#} \mathrm{p}<0.001\right.$ vs. Sham, $* \mathrm{p}<0.05$ vs. SAH treated with vehicle, $* * \mathrm{p}<0.01$ vs. SAH treated with vehicle)

\section{Effects of EP4 Receptor on the Expression of p-eNOS}

Western blot analysis showed Ser1177 p-eNOS expression increased $24 \mathrm{~h}$ after SAH (2.70 folds higher than sham group) (Fig. 6a, b). Compared with SAH treated with vehicle group, AE1-329 increased the expression of Ser1177 p-eNOS (3.94 folds higher than sham group) and AE3208 showed opposite effects (1.74 folds higher than sham group) (Fig. 6a, b).

\section{Discussion}

In this study we present the neuroprotective effects induced by EP4 receptor activation on EBI after SAH. Our results showed that the activation of EP4 by AE1-329 significantly improved neurological dysfunctions, reduced mortality, attenuated brain edema and BBB damage $24 \mathrm{~h}$ after SAH. In addition, EP4 activation downregulated the microglial activation, reduced the pro-inflammatory cytokine concentrations such as TNF- $\alpha$, IL- $1 \beta$ and IL- 6 in cortex and increased the Ser1177 p-eNOS expression $24 \mathrm{~h}$ after SAH. Moreover, EP4 activation decreased the number of TUNEL-positive cells and active caspase- 3 in cortex $24 \mathrm{~h}$ after SAH. These findings suggested that EP4 activation protected brain from SAH-induced EBI. The contrary effects induced by EP4 specific antagonist AE3-208 further contributed to determine the roles of EP4 in EBI after SAH.

The pathophysiologic mechanisms related to EBI are under investigating. Increased intracranial pressure (ICP), reduced cerebral blood flow $(\mathrm{CBF})$ and cerebral perfusion 


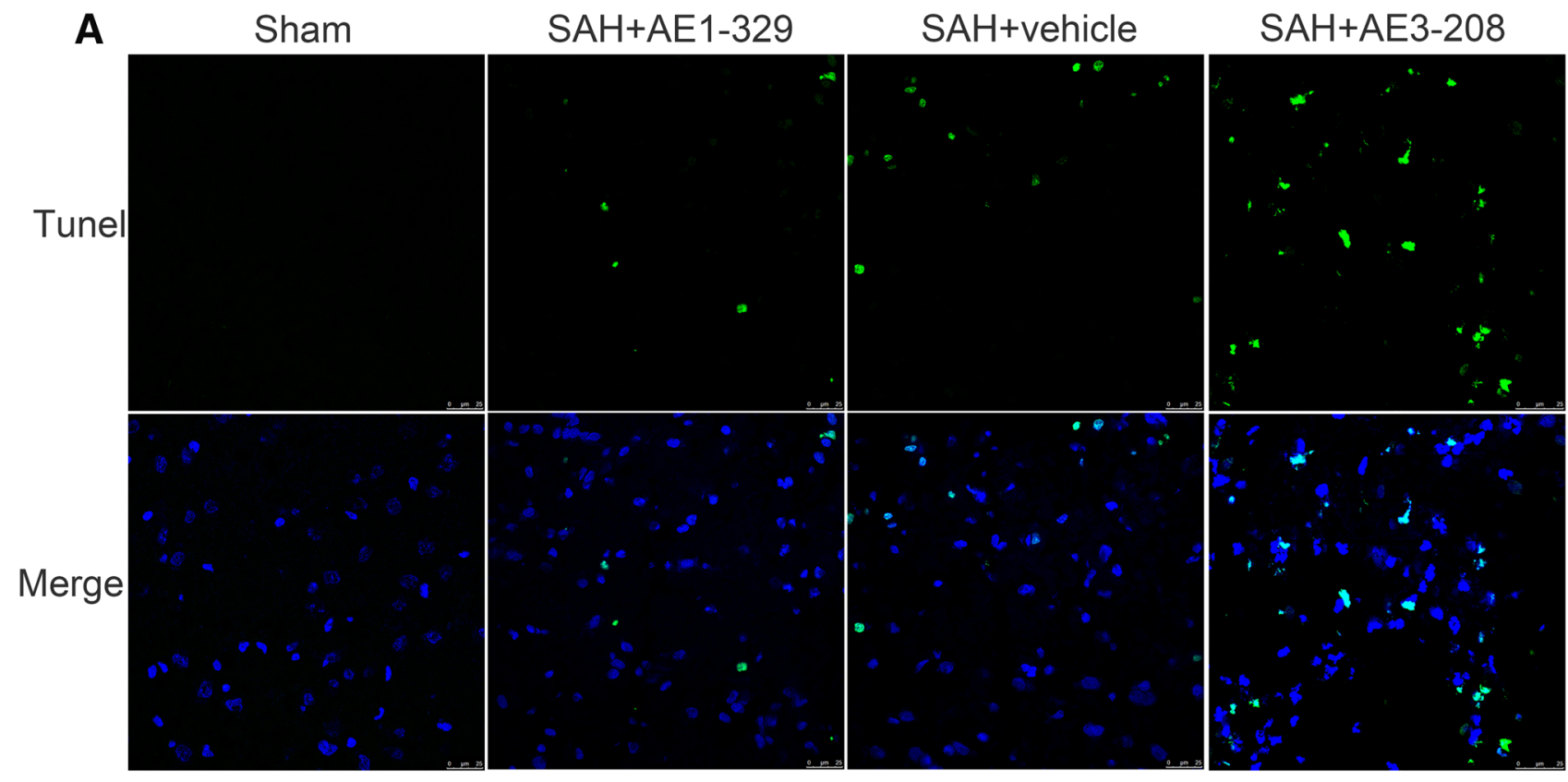

B

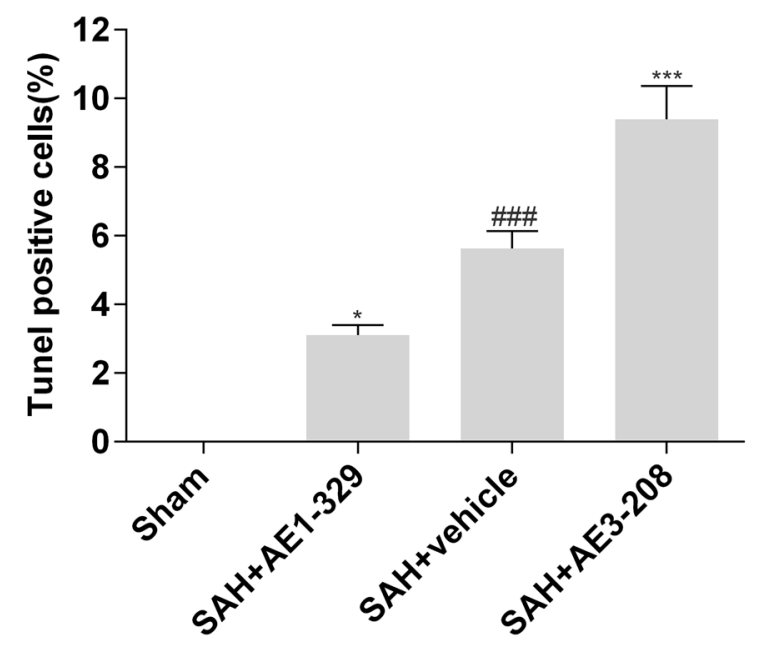

Fig. 3 Effects of EP4 receptor on neuronal apoptosis after SAH. TUNEL study showed the activation of EP4 by AE1-329 attenuated neuronal apoptosis $24 \mathrm{~h}$ after SAH while the inhibition of EP4 by AE3-208 showed opposite effects (a, b). Western blot analysis showed AE1-329 decreased the expression of cleaved caspase-3

pressure (CPP), BBB damage, brain edema, brain swelling and dysfunction of autoregulation, all of which stem from the initial bleeding in SAH, constitute the pathophysiological variables occurring at the EBI period [42]. Among them, the most immediate event following $\mathrm{SAH}$ is an arrest in intracranial circulation caused by a peak of ICP which can reach to the level of mean arterial blood pressure shortly after SAH [43]. This temporary circulatory arrest results in a hypoxic state which leads to the

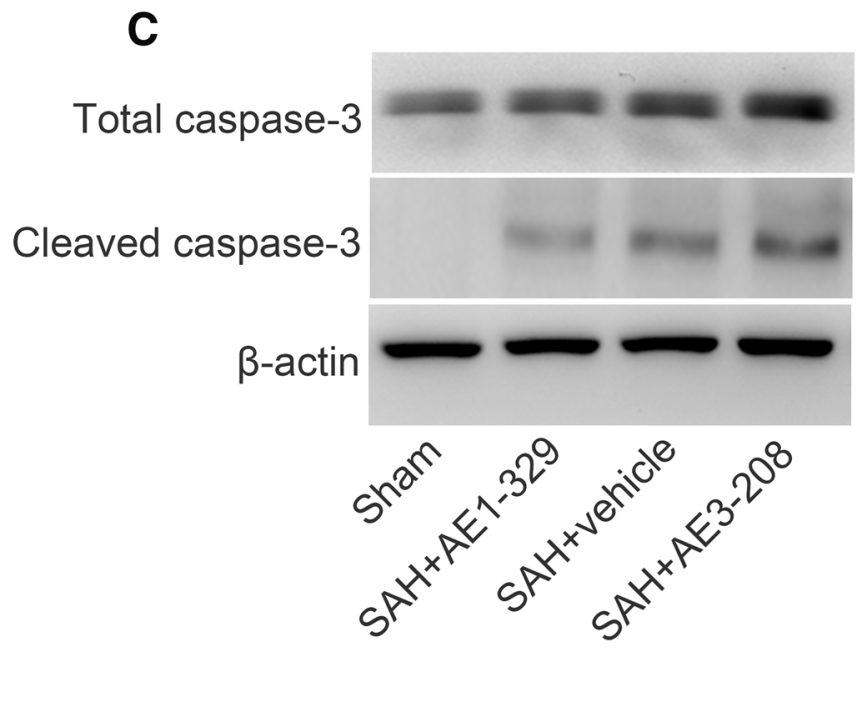

$24 \mathrm{~h}$ after SAH and AE3-208 showed opposite effects (c). Nuclear morphology was indicated by DAPI staining and DNA breaks were detected by TUNEL analyses. Bar: $25 \mu \mathrm{m}$. Data were expressed as mean \pm SEM $\left(n=8,{ }^{\# \# \# ~} p<0.001\right.$ vs. Sham, ${ }^{*} \mathrm{p}<0.05$ vs. SAH treated with vehicle, $* * * \mathrm{p}<0.001$ vs. SAH treated with vehicle)

apoptosis of neurons and glias due to the energy failure, and initiates the cascade of events leading to cytotoxic edema [42]. Ischemia also causes apoptosis of cells that constitute the BBB, which results in the increased diffusion of serum from the vascular lumen into cerebral tissues and eventually the vasogenic edema [44]. SAH also activates astrocytes and microglias, and upregulates the production and secretion of pro-inflammatory cytokines [45, 46]. Pro-inflammatory cytokines enhance brain 


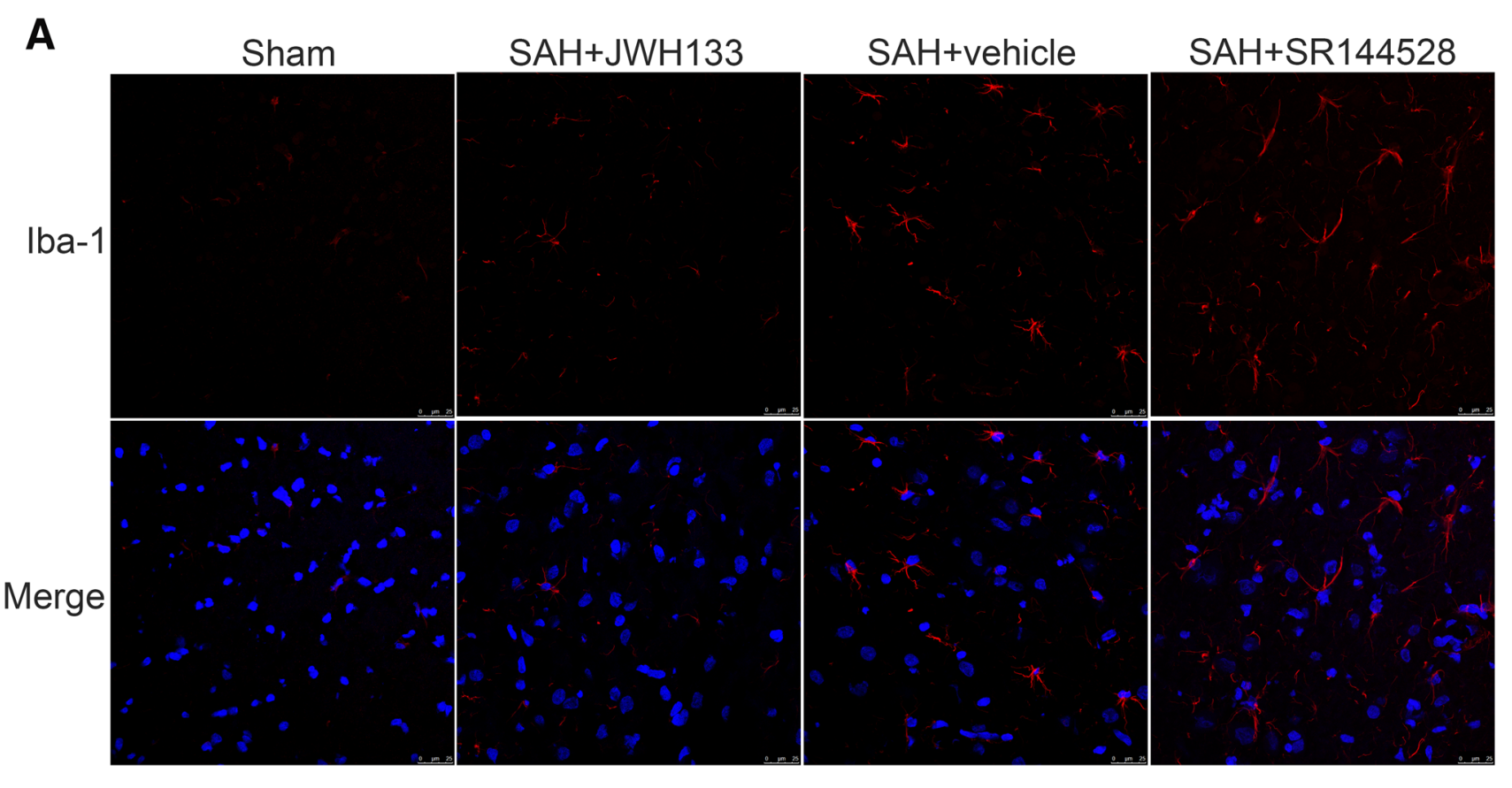

B

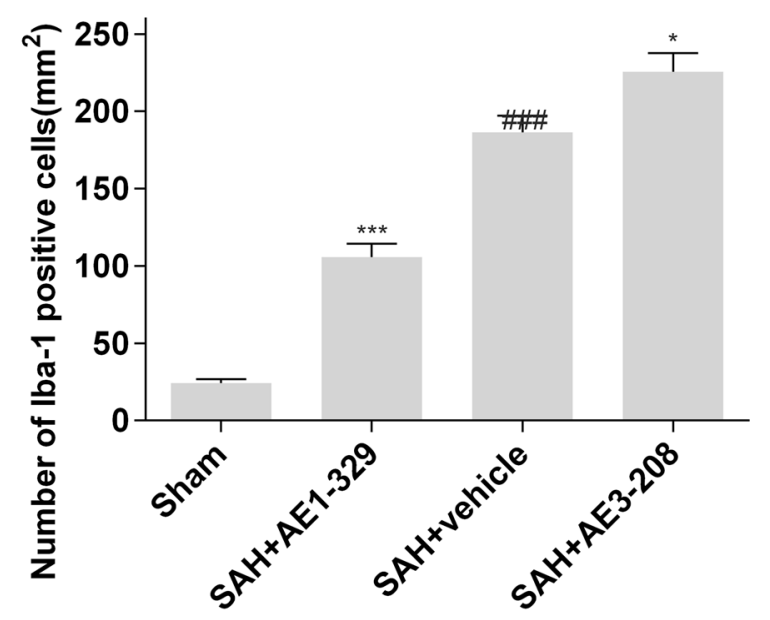

Fig. 4 Effects of EP4 receptor on the activation of microglia after SAH. Iba-1 staining for microglias showed activation of EP4 by AE1329 attenuated the number and activation of microglias in the brain $24 \mathrm{~h}$ after SAH while inhibition of EP4 by AE3-208 showed opposite

edema through the disrupting of BBB and induce neuronal apoptosis, and therefore directly contribute to early brain damage $[10,47]$.

Our data indicated that the activation of EP4 receptor by AE1-329 improved brain edema, BBB damage and neurological outcomes. These results are consistent with some previous studies demonstrating that the activation of EP4 receptor shows neuroprotective effects on other CNS insults such as cerebral ischemia [23, 24], hypoxic-ischemic effects (a, b). Microglia was indicated by Iba-1 staining and nuclear was indicated by DAPI staining. Bar: $25 \mu \mathrm{m}$. Data were expressed as

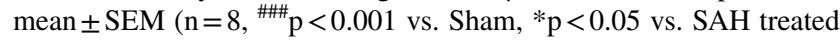
with vehicle, ${ }^{* * *} \mathrm{p}<0.001$ vs. SAH treated with vehicle)

encephalopathy (HIE) [25], neurotoxicity induced brain injury [26] and Alzheimer's disease [21].

The mechanisms underlying these EP4-induced neuroprotective effects were complicated and multiple cell types, including microglias, neurons and endothelial cells might be involved. Our findings indicated that the pharmacologic activation of EP4 receptor elicited the downregulation of microglial activity, the mRNA and protein expression of inflammatory cytokines. The production 


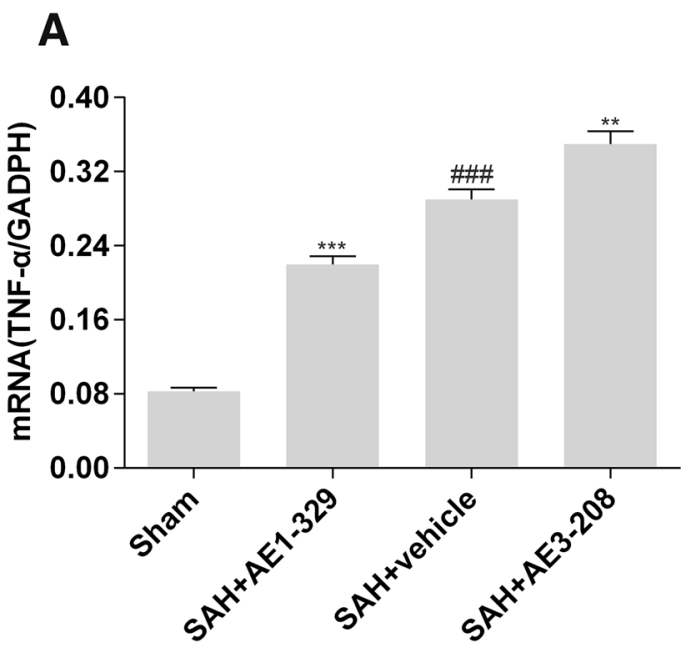

\section{B}

\section{C}
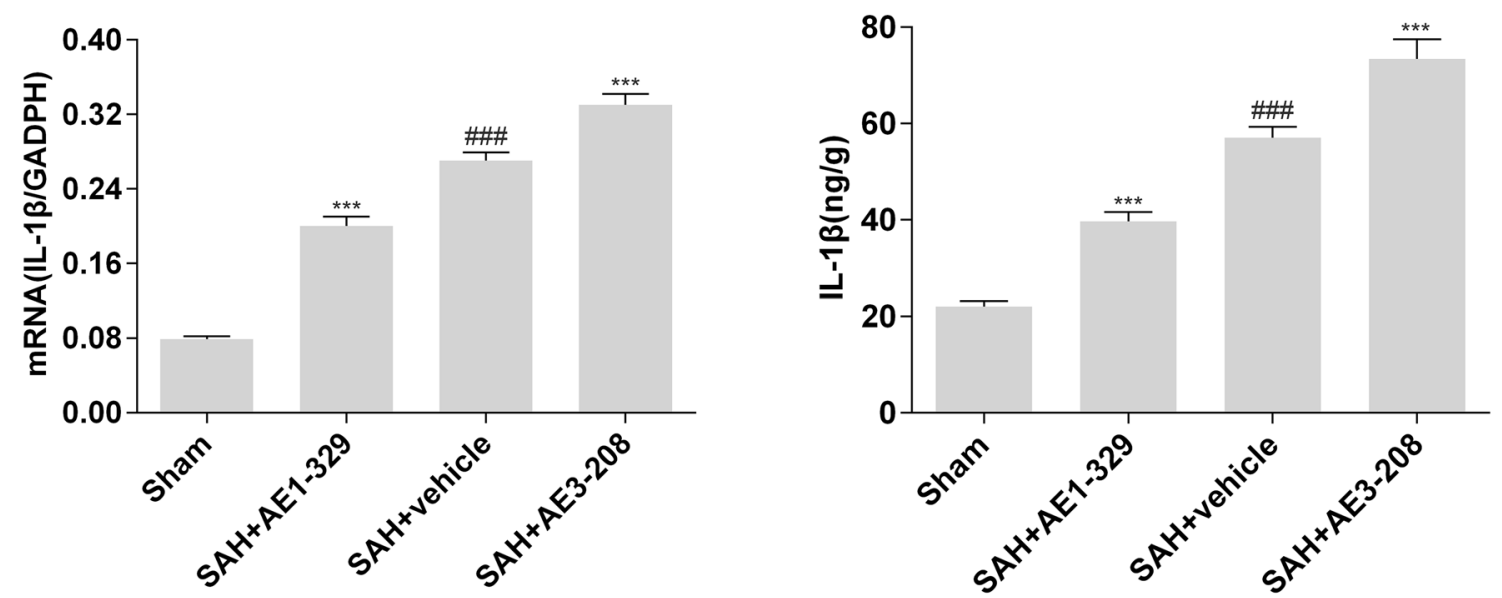

E

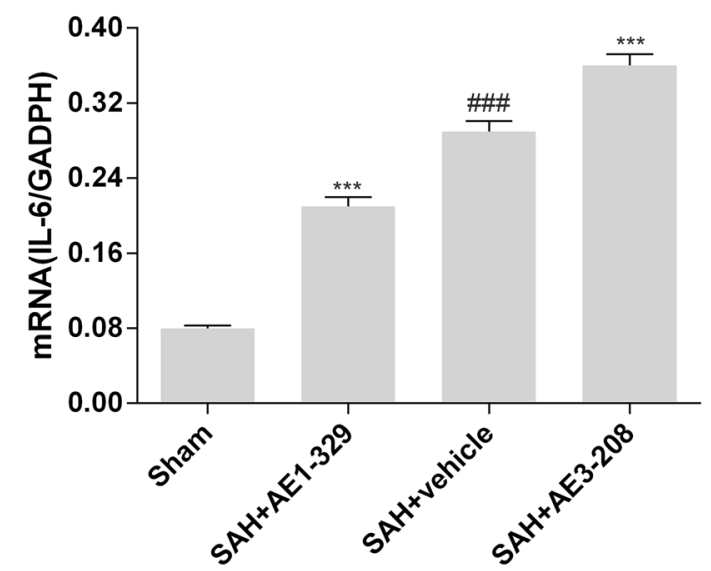

Fig. 5 Effects of EP4 receptor on the levels of inflammatory cytokines after SAH. RT-qPCR analysis showed EP4 activation by AE1-329 reduced the mRNA expression of TNF-a IL-1 $\beta$ and IL-6 while inhibition of EP4 by AE3-208 increased the mRNA expression $(\mathbf{a}, \mathbf{c}, \mathbf{e})$. Enzyme linked immunosorbent assay showed activation

$\mathbf{F}$

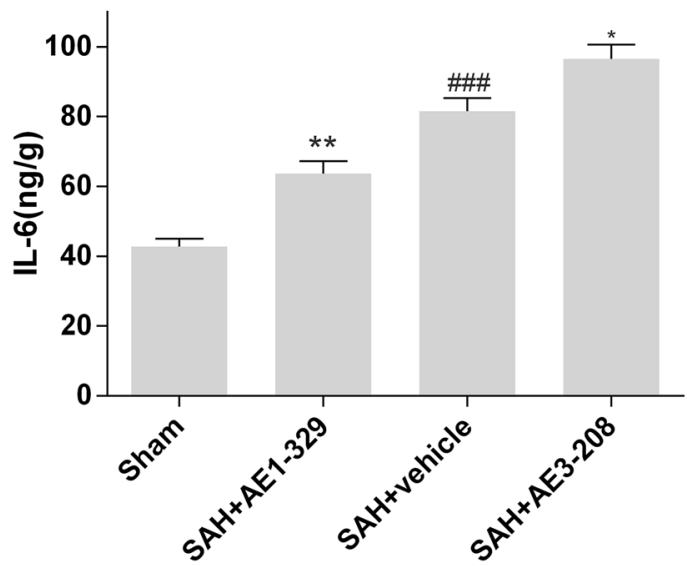

of EP4 by AE1-329 attenuated the levels of inflammatory cytokines while inhibition of EP4 by AE3-208 showed opposite effects (b, d, f). Data were expressed as mean \pm SEM ( $n=8,{ }^{\# \#} \mathrm{p}<0.001$ vs. Sham, $* p<0.05$ vs. SAH treated with vehicle, $* * p<0.01$ vs. SAH treated with vehicle, ${ }^{* * *} \mathrm{p}<0.001$ vs. SAH treated with vehicle) 
Fig. 6 Effects of EP4 receptor on the expression of p-eNOS. Western blot analysis showed AE1-329 increased the expression of Ser1177 p-eNOS $24 \mathrm{~h}$ after SAH and AE3-208 showed opposite effects $(\mathbf{a}, \mathbf{b})$. Data were expressed as mean \pm SEM $\left(\mathrm{n}=8,{ }^{\# \#} \mathrm{p}<0.001\right.$ vs. Sham, $* \mathrm{p}<0.05$ vs. SAH treated with vehicle, $* * \mathrm{p}<0.01$ vs. SAH treated with vehicle)
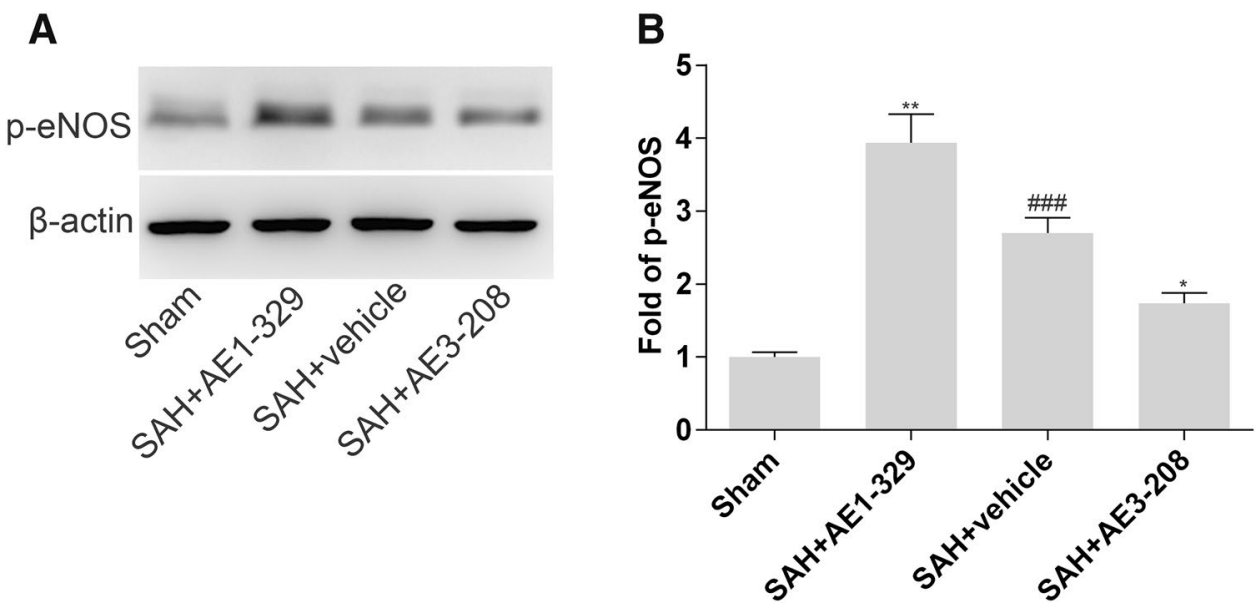

of pro-inflammatory cytokines is one of the hallmarks of microglial activation. In our study, we did not explored how SAH-induced production of TNF-a IL- $1 \beta$ and IL-6 was regulated by EP4 receptor. The suppression of microglial activation might account for the reduction of the pro-inflammatory cytokines. Going further, the activation of nuclear factor-kappaB (NF- $\mathrm{kB})$ is required for the generating of TNF-a, IL-1 $\beta$ and IL-6 in microglia [48]. Woodling et al. have found that EP4 signaling broadly represses the activation of target genes for interferon regulatory factors (IRFs) and NF- $\mathrm{KB}$, which are central transcription factors regulating the microglial response to $A \beta_{42}$ stimulation [21]. The similar NF- $\mathrm{KB}$ suppression after EP4 activation has also been reported by Shi et al. in a brain innate immunity model induced by lipopolysaccharide (LPS) [22].

In addition to anti-inflammatory effects, we also found the selective activation of EP4 significantly decreased the number of TUNEL positive cells and level of active caspase-3 after SAH. This should not be entirely ascribed to the suppression of neuroinflammatory reaction after EP4 activation, as EP4 receptors also locate on neurons and their activation by AE1-329 have shown a neuroprotective effect on brain ischemia [23].

We also found the upregulation of Ser1177 p-eNOS expression after AE1-329 administration. The endothelial EP4 receptor which expresses at a very low level in normal condition markedly upregulates in vasculature under the stimulation of ischemia and systemical administration of AE1-329 greatly elevate protein levels of eNOS and activated phospho-Ser1177 eNOS in mouse cerebral microvessels [23]. The activation of eNOS and increased NO production result in cGMP dependent smooth muscle cell relaxation and increased cerebral blood flow [49]. Endothelial EP4 activation by AE1-329 might directly inhibit the increase in BBB permeability in mouse experimental autoimmune encephalomyelitis [31].
Another neuroprotective mechanism initiated by EP4 activation in this study might involve the peripheral inflammatory cells including neutrophils, macrophages and lymphocytes. Transmission of inflammatory cells from periphery blood to CNS has been demonstrated in the clinical and animal studies of SAH, which can result in not only the neuroinflammatory aggravation but also the early neuronal loss and BBB damage $[50,51]$. Peripheral administration of the EP4 specific agonist AE1-329 downregulates the secreted levels of pro-inflammatory cytokines and chemokines from macrophages and neutrophils [22, 52].

Taken together, in present study, EP4 activation appears to have a multiple beneficial properties for EBI after SAH. However, the precise mechanisms of the neuroprotective effects of EP4 activation on EBI after SAH remain unclear and further studies exploring the underlying mechanisms of $\mathrm{EP} 4$ are required in future.

\section{Conclusion}

This study is the first one to explore the effects of EP4 receptor activation on EBI after experimental SAH. We find the activation of EP4 receptor by AE1-329 improves neurological dysfunctions, and ameliorates brain edema and BBB damages after SAH. Meanwhile activation of EP4 receptor suppresses microglial activation, the protein and mRNA levels of pro-inflammatory cytokines TNF-a, IL- $1 \beta$ and IL- 6 and increases Ser1177 p-eNOS expression. Furthermore, activation of EP4 receptor attenuates cellular apoptosis through downregulating active caspase-3. Our results suggest EP4 receptor may be a promising potential target for SAH therapy.

Acknowledgements This work was supported by Zhejiang Provincial Medical Health and Science and Technology Project Foundation (2015KYB375). 
Authors' Contributions Zhen $\mathrm{Xu}$ and Jie $\mathrm{Xu}$ designed the experiments and prepared the models. Ai Yan prepared the SAH models and carried out the experiments.

\section{Compliance with Ethical Standards}

Conflict of interest The authors declare no conflict of interest.

Open Access This article is distributed under the terms of the Creative Commons Attribution 4.0 International License (http:// creativecommons.org/licenses/by/4.0/), which permits unrestricted use, distribution, and reproduction in any medium, provided you give appropriate credit to the original author(s) and the source, provide a link to the Creative Commons license, and indicate if changes were made.

\section{References}

1. Macdonald RL (2014) Delayed neurological deterioration after subarachnoid haemorrhage. Nat. Rev Neurol 10:44-58

2. Wilkins RH (1990) Cerebral vasospasm. Crit Rev Neurobiol 6:51-77

3. Dorsch NW (1995) Cerebral arterial spasm-a clinical review. Br J Neurosurg 9:403-412

4. Velat GJ, Kimball MM, Mocco JD, Hoh BL (2011) Vasospasm after aneurysmal subarachnoid hemorrhage: review of randomized controlled trials and meta-analyses in the literature. World Neurosurg 76:446-454.

5. Sehba FA, Hou J, Pluta RM, Zhang JH (2012) The importance of early brain injury after subarachnoid hemorrhage. Prog Neurobiol 97:14-37

6. Kusaka G, Ishikawa M, Nanda A, Granger DN, Zhang JH (2004) Signaling pathways for early brain injury after subarachnoid hemorrhage. J Cereb Blood Flow Metab 24:916-925

7. Pluta RM, Hansen-Schwartz J, Dreier J, Vajkoczy P, Macdonald RL, Nishizawa S, Kasuya H, Wellman G, Keller E, Zauner A, Dorsch N, Clark J, Ono S, Kiris T, Leroux P, Zhang JH (2009) Cerebral vasospasm following subarachnoid hemorrhage: time for a new world of thought. Neurol Res 31:151-158

8. Suzuki H, Hasegawa Y, Chen W, Kanamaru K, Zhang JH (2010) Recombinant osteopontin in cerebral vasospasm after subarachnoid hemorrhage. Ann Neurol 68:650-660

9. Sherchan P, Lekic T, Suzuki H, Hasegawa Y, Rolland W, Duris K, Zhan Y, Tang J, Zhang JH (2011) Minocycline improves functional outcomes, memory deficits, and histopathology after endovascular perforation-induced subarachnoid hemorrhage in rats. J Neurotrauma 28:2503-2512

10. Sozen T, Tsuchiyama R, Hasegawa Y, Suzuki H, Jadhav V, Nishizawa S, Zhang JH (2009) Role of interleukin-1beta in early brain injury after subarachnoid hemorrhage in mice. Stroke 40:2519-2525

11. Sugawara T, Jadhav V, Ayer R, Chen W, Suzuki H, Zhang JH (2009) Thrombin inhibition by argatroban ameliorates early brain injury and improves neurological outcomes after experimental subarachnoid hemorrhage in rats 40:1530-1532.

12. Erşahin M, Toklu HZ, Erzik C, Cetinel S, Akakin D, VelioğluOğünç A, Tetik S, Ozdemir ZN, Sener G, Yeğen BC (2010) The anti-inflammatory and neuroprotective effects of ghrelin in subarachnoid hemorrhage-induced oxidative brain damage in rats. $\mathbf{J}$ Neurotrauma 27:1143-1155

13. Sivakumar V, Foulds WS, Luu CD, Ling EA, Kaur C (2011) Retinal ganglion cell death is induced by microglia derived pro-inflammatory cytokines in the hypoxic neonatal retina. $\mathrm{J}$ Pathol 224:245-260

14. Gaetani P, Tartara F, Pignatti P, Tancioni F, Rodriguez y Baena R, De Benedetti F (1998) Cisternal CSF levels of cytokines after subarachnoid hemorrhage. Neurol Res 20:337-342

15. Greenhalgh AD, Brough D, Robinson EM, Girard S, Rothwell NJ, Allan SM (2012) Interleukin-1 receptor antagonist is beneficial after subarachnoid haemorrhage in rat by blocking haemdriven inflammatory pathology. Dis Model Mech 5:823-833

16. Larysz-Brysz M, Lewin-Kowalik J, Czuba Z, Kotulska K, Olakowska E, Marcol W, Liśkiewicz A, Jędrzejowska-Szypułka $H$ (2012) Interleukin-1 $\beta$ increases release of endothelin-1 and tumor necrosis factor as well as reactive oxygen species by peripheral leukocytes during experimental subarachnoid hemorrhage. Curr Neurovasc Res 9:159-166

17. Ganesh T (2014) Prostanoid receptor EP2 as a therapeutic target. J Med Chem 57:4454-4465

18. Breyer RM, Bagdassarian CK, Myers SA, Breyer MD (2001) Prostanoid receptors: subtypes and signaling. Annu Rev Pharmacol Toxicol 41:661-690

19. Negishi M, Sugimoto Y, Ichikawa A (1995) Prostaglandin E receptors. J Lipid Mediat Cell Signal 12:379-391

20. Konya V, Marsche G, Schuligoi R, Heinemann A (2013) E-type prostanoid receptor 4 (EP4) in disease and therapy. Pharmacol Ther 138:485-502

21. Woodling NS, Wang Q, Priyam PG, Larkin P, Shi J, Johansson JU, Zagol-Ikapitte I, Boutaud O, Andreasson KI (2014) Suppression of Alzheimer-associated inflammation by microglial prostaglandin-E2 EP4 receptor signaling. J Neurosci 34:5882-5894

22. Shi J, Johansson J, Woodling NS, Wang Q, Montine TJ, Andreasson K (2010) The prostaglandin E2 E-prostanoid 4 receptor exerts anti-inflammatory effects in brain innate immunity. J Immunol 184:7207-7218

23. Liang X, Lin L, Woodling NS, Wang Q, Anacker C, Pan T, Merchant M, Andreasson K (2011) Signaling via the prostaglandin E2 receptor EP4 exerts neuronal and vascular protection in a mouse model of cerebral ischemia. J Clin Invest 121:4362-4371

24. Akram A, Gibson CL, Grubb BD (2013) Neuroprotection mediated by the EP4 receptor avoids the detrimental side effects of COX-2 inhibitors following ischaemic injury. Neuropharmacology $65: 165-172$

25. Taniguchi H, Anacker C, Wang Q, Andreasson K (2014) Protection by vascular prostaglandin E2 signaling in hypoxic-ischemic encephalopathy. Exp Neurol 255:30-37

26. Ahmad AS, Ahmad M, de Brum-Fernandes AJ, Doré S (2005) Prostaglandin EP4 receptor agonist protects against acute neurotoxicity. Brain Res 1066:71-77

27. Sugawara T, Ayer R, Jadhav V, Zhang JH (2008) A new grading system evaluating bleeding scale in filament perforation subarachnoid hemorrhage rat model. J Neurosci Methods 167:327-334

28. Zhang ZY, Sun BL, Liu JK, Yang MF, Li DW, Fang J, Zhang S, Yuan QL, Huang SL (2015) Activation of mGluR5 attenuates microglial activation and neuronal apoptosis in early brain injury after experimental subarachnoid hemorrhage in rats. Neurochem Res 40:1121-1132

29. Suzawa T, Miyaura C, Inada M, Maruyama T, Sugimoto $Y$, Ushikubi F, Ichikawa A, Narumiya S, Suda T (2000) The role of prostaglandin E receptor subtypes (EP1, EP2, EP3, and EP4) in bone resorption: an analysis using specific agonists for the respective EPs. Endocrinology 141:1554-1559

30. Kabashima K, Saji T, Murata T, Nagamachi M, Matsuoka T, Segi E, Tsuboi K, Sugimoto Y, Kobayashi T, Miyachi Y, Ichikawa A, Narumiya S (2002) The prostaglandin receptor EP4 suppresses colitis, mucosal damage and CD4 cell activation in the gut. J Clin Invest 109:883-893 
31. Esaki Y, Li Y, Sakata D, Yao C, Segi-Nishida E, Matsuoka T, Fukuda K, Narumiya S (2010) Dual roles of PGE2-EP4 signaling in mouse experimental autoimmune encephalomyelitis. Proc Natl Acad Sci U S A 107:12233-12238

32. Hoshino $T$, Namba $T$, Takehara M, Murao N, Matsushima T, Sugimoto Y, Narumiya S, Suzuki T, Mizushima T (2012) Improvement of cognitive function in Alzheimer's disease model mice by genetic and pharmacological inhibition of the EP(4) receptor. J Neurochem 120:795-805

33. Sugawara T, Ayer R, Jadhav V, Chen W, Tsubokawa T, Zhang JH (2008) Simvastatin attenuation of cerebral vasospasm after subarachnoid hemorrhage in rats via increased phosphorylation of Akt and endothelial nitric oxide synthase. J Neurosci Res 86:3635-3643

34. Hasegawa Y, Suzuki H, Altay O, Zhang JH (2011) Preservation of tropomyosin-related kinase B (TrkB) signaling by sodium orthovanadate attenuates early brain injury after subarachnoid hemorrhage in rats. Stroke 42:477-483

35. Garcia JH, Wagner S, Liu KF, Hu XJ (1995) Neurological deficit and extent of neuronal necrosis attributable to middle cerebral artery occlusion in rats. Statistical validation. Stroke 26:627-634

36. Roof RL, Duvdevani R, Heyburn JW, Stein DG (1996) Progesterone rapidly decreases brain edema: treatment delayed up to $24 \mathrm{~h}$ is still effective. Exp Neurol 138:246-251

37. Fujii M, Duris K, Altay O, Soejima Y, Sherchan P, Zhang JH (2012) Inhibition of Rho kinase by hydroxyfasudil attenuates brain edema after subarachnoid hemorrhage in rats. Neurochem Int 60:327-333

38. Wang JW, Wang HD, Cong ZX, Zhang XS, Zhou XM, Zhang DD (2013) Activation of metabotropic glutamate receptor 5 reduces the secondary brain injury after traumatic brain injury in rats. Biochem Biophys Res Commun 430:1016-1021

39. Hanisch UK, Kettenmann H (2007) Microglia: active sensor and versatile effector cells in the normal and pathologic brain. Nat Neurosci 10:1387-1394

40. Yuskaitis CJ, Jope RS (2009) Glycogen synthase kinase-3 regulates microglial migration, inflammation, and inflammationinduced neurotoxicity. Cell Signal 21:264-273

41. Farfara D, Lifshitz V, Frenkel D (2008) Neuroprotective and neurotoxic properties of glial cells in the pathogenesis of Alzheimer's disease. J Cell Mol Med 12:762-780
42. Ostrowski RP, Colohan AR, Zhang JH (2006) Molecular mechanisms of early brain injury after subarachnoid hemorrhage. Neurol Res 28:399-414.

43. Fujii M, Yan J, Rolland WB, Soejima Y, Caner B, Zhang JH (2013) Early brain injury, an evolving frontier in subarachnoid hemorrhage research. Transl Stroke Res 4:432-446

44. Keep RF, Andjelkovic AV, Stamatovic SM, Shakui P, Ennis SR (2005) Ischemia-induced endothelial cell dysfunction. Acta Neurochir Suppl 95:399-402

45. Murakami K, Koide M, Dumont TM, Russell SR, Tranmer BI, Wellman GC (2011) Subarachnoid hemorrhage induces gliosis and increased expression of the pro-inflammatory cytokine high mobility group box 1 protein. Transl Stroke Res 2:72-79

46. Prunell GF, Svendgaard NA, Alkass K, Mathiesen T (2005) Inflammation in the brain after experimental subarachnoid hemorrhage. Neurosurgery 56:1082-1092

47. Edvinsson L, Larsen SS, Maddahi A, Nielsen J (2014) Plasticity of cerebrovascular smooth muscle cells after subarachnoid hemorrhage. Transl Stroke Res 5:365-376

48. You WC, Wang CX, Pan YX, Zhang X, Zhou XM, Zhang XS, Shi JX, Zhou ML (2013) Activation of nuclear factor- $\kappa B$ in the brain after experimental subarachnoid hemorrhage and its potential role in delayed brain injury. PLoS One 8:e60290

49. Endres M, Laufs U, Liao JK, Moskowitz MA (2004) Targeting eNOS for stroke protection. Trends Neurosci 27:283-289

50. Moraes L, Grille S, Morelli P, Mila R, Trias N, Brugnini A, LLuberas N, Biestro A, Lens D (2015) Immune cells subpopulations in cerebrospinal fluid and peripheral blood of patients with aneurysmal subarachnoid hemorrhage. Springerplus 4:195.

51. Mracsko E, Javidi E, Na SY, Kahn A, Liesz A, Veltkamp R (2014) Leukocyte invasion of the brain after experimental intracerebral hemorrhage in mice 45:2107-2114.

52. Yamane H, Sugimoto Y, Tanaka S, Ichikawa A (2000) Prostaglandin E(2) receptors, EP2 and EP4, differentially modulate TNF-alpha and IL-6 production induced by lipopolysaccharide in mouse peritoneal neutrophils. Biochem Biophys Res Commun 278:224-228 